\title{
Capsule Commentary on Young et al., Effects of Physician Experience, Specialty Training, and Self Referral on Inappropriate Diagnostic Imaging
}

\author{
Kia Smurawa, BS
}

Medical College of Wisconsin, Milwaukee, WI, USA.

J Gen Intern Med 35(6): 1935

DOI: $10.1007 / \mathrm{s} 11606-020-05706-4$

(c) Society of General Internal Medicine 2020

$\mathrm{H}$ ealth care spending in the USA is disproportionately high compared with other high-income countries. The USA is the largest user of CT scans and the second largest user of MRIs, both of which significantly contribute to overall health care costs. ${ }^{1}$ As Young et al. ${ }^{2}$ suggest, a substantial portion of diagnostic image testing is inappropriate; reducing inappropriate diagnostic imaging could reduce overall spending.

To explore a potential source for this problem, Young et al. examined the influence of physician experience and specialization on inappropriate diagnostic imaging. Based on appropriateness criteria from the American College of Radiology (ACR) and claims from the Massachusetts All-Payer Claims database, they determined that $23 \%$ of MRI referrals for lower back pain, non-traumatic knee pain, and non-traumatic shoulder pain were inappropriate. Physicians with 10 years of training or less had the highest percent of inappropriate imaging (28\%). Primary Care specialties were more likely to order an unnecessary MRI than Orthopedic specialists. There was no correlation between self-referral and inappropriate imaging. Additionally, patients with HMO insurance plans were less likely to receive inappropriate imaging, likely due to heightened pre-authorization review.

A strength of this study is the use of ACR criteria, though with claims data it is impossible to determine if the physicians knowingly ordered inappropriate images. A 2015 study found that most Emergency physicians believed patients in their specialty received unnecessary diagnostic tests and admitted to performing them themselves. ${ }^{3}$ Other studies suggest that fear of malpractice is the biggest driving factor in over-testing, followed by patient pressure. ${ }^{4}$ Rather than clinical inexperience, heightened fear of malpractice suits may explain certain groups over-imaging. While Young suggests that education on imaging guidelines may reduce inappropriate imaging, if physicians are knowingly ordering inappropriate images, such training would be ineffective. More research must be done to determine how often clinicians knowingly order inappropriate images and the underlying motivation, whether clinical inexperience, inadequate knowledge of imaging criteria, or fear of malpractice. Once this is better understood, appropriate interventional trials can be designed to improve imaging practices.

Corresponding Author: Kia Smurawa, BS; Medical College of Wisconsin, Milwaukee, WI, USA (e-mail: ksmurawa@mcw.edu).

Compliance with Ethical Standards:

Conflict of Interest: The author has no conflicts of interest with this article.

\section{REFERENCES}

1. Emanuel EJ. The Real Cost of the US Health Care System. JAMA. 2018:319(10):983-985. https://doi.org/10.1001/jama.2018.1151

2. Young GJ, Flaherty S, Zepeda ED, Mortele K, Griffith J. Effects of Physician Experience, Specialty Training, and Self Referral on Inappropriate Diagnostic Imaging $\mathrm{J}$ Gen Intern Med. https://doi.org/10.1007/ s11606-019-05621-3.

3. Kanzaria HK, Hoffman JR, Probst MA, Caloyeras JP, Berry SH, Brook RH (2015). Emergency Physician Perceptions of Medically Unnecessary Advanced Diagnostic Imaging. Acad Emerg Med, 22(4), 390-398. https:// doi.org/10.1111/acem. 12625

4. Lyu H, Xu T, Brotman D, Mayer-Blackwell B, Cooper M, Daniel M, et al. Overtreatment in the United States. PLoS One 2017;12(9):e0181970 https://doi.org/10.1371/journal.pone.0181970

Publisher's Note Springer Nature remains neutral with regard to jurisdictional claims in published maps and institutional affiliations.

Published online February 19, 2020 